\title{
Comparison Between Combined Sensory Index Test and Diagnostic Ultrasonography (Inlet Outlet Ratio) in Suspected or Early Cases of Carpal Tunnel Syndrome
}

Mennatallah M. Mustafa, Nagwa M. Nassar, Iren R. Amin, Hossam S. Labib*

Department of Physical Medicine, Rheumatology and Rehabilitation, Ain Shams University Hospital, Cairo, Egypt

*Corresponding author: Hossam Salaheldin Abdelmohsen Labib, Mobile: (+20)1002058687,

Email: hossam_salah@med.asu.edu.eg

\begin{abstract}
Background: The combined sensory index (CSI) test is more precise in diagnosing carpal tunnel syndrome (CTS) early cases. Another useful tool for early detection of CTS is the diagnostic ultrasonography.

Objective: The present study was conducted to compare between the CSI test and its sensitivity with diagnostic ultrasonography IOR (inlet outlet ratio) in suspected or early cases of carpal tunnel syndrome (CTS).

Patients and methods: The present case-control study involved 20 subjects with signs and symptoms suggestive of early cases of CTS with duration less than 6 months, in addition to 20 apparently healthy subjects who were clinically examined and underwent EDX and US evaluation. We excluded patients with severe CTS, proximal cervical lesion, or other neurological diseases.

Results: By comparison, CSI shows a higher sensitivity than IOR. Combining both tests induced elevated substantial differences between patients as well as controls ( $\mathrm{P}<0.01)$, besides elevating the sensitivity to $100 \%$.

Conclusion: It could be concluded that the sensitivity and accuracy of CSI is higher than diagnostic ultrasonography IOR on the median nerve. On the contrary, diagnostic ultrasonography can detect the anatomical abnormalities of the median nerve while the physiological abnormalities of the median nerve and their level can be examined by nerve conduction studies (NCS). They are complementary tests for CTS diagnosis
\end{abstract}

Keywords: Combined sensory index, Carpal tunnel syndrome, Diagnostic ultrasonography IOR (inlet outlet ratio)

\section{INTRODUCTION}

Carpal tunnel syndrome (CTS) is a prevalent form of nerve compression syndromes. It is induced by entrapment of the median nerve at the wrist and occurs in approximately $3.8 \%$ of people ${ }^{(\mathbf{1})}$. According to the guidelines, the diagnose of CTS includes clinical finding and electro diagnostic test ${ }^{(2)}$.

In early stages, clinical finding is insufficient for CTS diagnosis as the history and topographic distribution of symptoms are nearly normal. In these suspected cases, combined sensory index (CSI) which is the aggregate of average ulnar peak sensory latency difference (Ring difference), average radial peak sensory latency difference (Thumb difference), as well as average ulnar mid palmer peak sensory latency difference (Palm difference) are considered more sensitive ${ }^{(3)}$.

Another useful tool for early detection of CTS is diagnostic ultrasonography which is utilized to evaluate median nerve anatomical abnormalities that NCS cannot evaluate. The importance of ultrasonography is manifested in its vast availability, noninvasiveness, reduced cost as well as decreased time of examination (4). The most widely method used to diagnosis CTS by ultrasonography is measuring the median curve crosssectional area (CSA) at the wrist on two regions (outlet as well as inlet of the tunnel). The proportion between these two regions namely, Inlet _outlet ratio (IOR) has recently been used to compensate for the individual inter variability in the median nerve CSA and to give a more precise CTS diagnosis ${ }^{(5)}$.
The aim of the current work was to compare between the CSI test and its sensitivity with diagnostic ultrasonography IOR (inlet outlet ratio) in suspected or early cases of carpal tunnel syndrome (CTS).

\section{PATIENTS AND METHODS}

This case-control study included a total of 20 participants who developed signs and symptoms suggestive of early cases of CTS with duration less than 6 months, in addition to 20 apparently healthy subjects as controls, attending at Outpatient Clinics, Department of Physical Medicine, Rheumatology and Rehabilitation, Ain Shams University Hospitals, Cairo, Egypt.

\section{Ethical Consideration:}

An approval of the study was obtained from Ain Shams University academic and ethical committee. Every patient signed an informed written consent for acceptance of the operation. This work has been carried out in accordance with The Code of Ethics of the World Medical Association (Declaration of Helsinki) for studies involving humans.

Inclusion Criteria: Cases which developed signs and suggestive CTS symptoms ${ }^{(4)}$ Nocturnal paresthesia in lateral three and half fingers, hand shaking (the flick sign) alleviates numbness sensation and tingling, burning pain in the in lateral three and half fingers, positive Phalen's test, positive Hoffman-tinel sign and positive compression test. 
Exclusion criteria: Depend on history and clinical examination. Criteria include any proximal cervical lesion, peripheral polyneuropathy or any other neurological diseases, patients with severe CTS.

\section{All patients underwent the following:}

- Complete medical history.

- Extensive clinical screening.

- General examination.

- Local examination and wrist were assessed by provocative tests.

- Nerve conduction studies (NCS).

All NCS were performed using TOPAS SHWARZER version 1.59 EMG machine. The patient was placed in sitting position, with the forearm supine. A complete explanation of the procedure was conveyed to the patient.

\section{The following indices were obtained:}

Distal motor latency as well as f-wave for both ulnar and median nerves in order to exclude any other CTS inducers.

Thumb difference (Median-Radial Latency Difference to the Thumb): Median as well as radial nerve stimulation antidromic technique at the wrist via ring electrodes on the thumb with a distance of $10 \mathrm{~cm}$ from the stimulus to the recording.

Ring difference test (the difference of median-ulnar sensory latency to ring finger): Antidromic technique for stimulating ulnar and median nerves at the wrist as well as recording $14 \mathrm{~cm}$ from the ring finger using ring electrodes.

Palm difference (mid-ulnar mid-palmar difference test): Orthodromic technique stimulation is performed in the palm over the median as well as ulnar nerve stimulation along its path in the palm, $8 \mathrm{~cm}$ away from the active recording electrode.

Combined sensory index test: The sum of: (Thumb difference + Ring difference + Palm difference) .

\section{Diagnostic ultrasonography (US):}

U.S evaluation of the wrist was conducted via a highfrequency (0-12 MHZ) linear transducer (LOGIQ 500 pro series, GE medical systems, USA). Patients were seated on a comfortable chair with the elbow extended while hands rested in a supine horizontal position on the examination bed with semi-extended fingers. US scans (transverse as well as longitudinal scans) of the median nerve were done from the forearm distal part to the outlet of the tunnel. Subsequently, the measurement of the median nerve CSA was carried out:

- At inlet of the tunnel (before the near margin of the flexor retinaculum).

- At the outlet of tunnel (at the level of hamate hook).
- Inlet-outlet ratio (IOR): estimated through dividing the median nerve CSA at inlet of the tunnel over its CSA at the outlet of the tunnel.

\section{Statistical analysis}

Was performed using the median, student's t-test, standard deviation, chi-square, as well as the linear correlation coefficient tests by SPSS V17. The ranges of means and standard deviations were utilized in order to summarize the features of samples. Summarization of categorical variables was using frequencies and percentages. Unpaired Student T-test was utilized to make a comparison between both groups in quantitative data. Moreover, the linear Correlation coefficient was utilized to determine the association between two quantitative variables in the same group. The calculation of ROC curves was done to determine the specificity, sensitivity and accuracy of the NCS and US tests. The $\mathrm{P}$ value was considered significant when it is less than 0.05 .

\section{RESULTS}

The present case-control study involved 20 subjects with symptoms and signs suggestive of early cases of CTS with a duration less than 6 months and twenty apparently healthy volunteers as controls. No substantial differences were detected between both groups with respect to age and gender.

Based on history, all patients suffered from paresthesia in three lateral and half fingers as well as nocturnal paresthesia. On examination using provocative tests, there was positive Tinel teste in 8 patients, positive Phalen's test in 12 patients with and positive compression in 12 patients with percentage $60 \%$, as demonstrated in (Table 1).

Table (1): Description of the clinical data of the patients group:

\begin{tabular}{|l|c|c|}
\hline \multicolumn{1}{|c|}{$\begin{array}{c}\text { Clinical diagnostic } \\
\text { parameters }\end{array}$} & N of positive & $\%$ \\
\hline $\begin{array}{l}\text { Paresthesia in lateral 3 } \\
\text { fingers }\end{array}$ & 20 & 100 \\
\hline Nocturnal paresthesia & 20 & 100 \\
\hline Tinel Test & 8 & 40 \\
\hline Phalen Test & 12 & 60 \\
\hline Compression Test & 12 & 60 \\
\hline
\end{tabular}

Electrodiagnostic tests among the control as well as the patient groups:

Comparison between controls as well as patients regarding CSI test, thumb difference test, and ring difference test demonstrated substantial differences between both groups $(\mathrm{P}<0.001)$. While palm difference test demonstrated non-significant differences between both groups ( $\mathrm{P}>0.05)$, as displayed in Table (2). 
Table (2): The electrodiagnostic sensory peak latency difference between controls and patient group.

\begin{tabular}{|l|c|c|c|c|}
\hline & $\begin{array}{c}\text { Patients with CTS } \\
(\text { mean } \pm \text { SD) }\end{array}$ & $\begin{array}{c}\text { The control } \\
(\mathbf{m e a n} \pm \text { SD) }\end{array}$ & T & P-value \\
\hline Thumb-difference (ms) & $0.61 \pm 0.36$ & $0.2 \pm 0.12$ & 4.813 & $<0.001$ \\
\hline Ring-difference (ms) & $0.44 \pm 0.28$ & $0.17 \pm 0.109$ & 4.025 & $<0.001$ \\
\hline Palm-difference (ms) & $0.305 \pm 0.327$ & $0.23 \pm 0.133$ & 1.014 & 1.014 \\
\hline CSI & $1.36 \pm 0.366$ & $0.59 \pm 0.187$ & 8.371 & $<0.001$ \\
\hline
\end{tabular}

Diagnostic ultrasonography among patient and control groups:

As regard median nerve CSA at the inlet of the tunnel and the IOR, it revealed highly substantial differences between the patient and control groups ( $\mathrm{P}<0.001)$. While CSA of medial nerve at tunnel outlet demonstrated non-significant differences between both groups $(\mathrm{P}>0.05)$, as illustrated in Table (3).

Table (3): The Diagnostic ultrasonography among patient and control groups

\begin{tabular}{|l|c|c|c|c|}
\hline & $\begin{array}{c}\text { Patients with CTS } \\
(\mathbf{m e a n} \pm \mathbf{S D})\end{array}$ & $\begin{array}{c}\text { The control } \\
(\mathbf{m e a n} \pm \mathbf{S D})\end{array}$ & T & P-value \\
\hline $\begin{array}{l}\text { Ultrasound CSA } \\
\text { inlet }\left(\mathbf{m m}^{2}\right)\end{array}$ & $9.7 \pm 1.55$ & $7.15 \pm 1.387$ & 5.464 & $<0.001$ \\
\hline $\begin{array}{l}\text { Ultrasound CSA } \\
\text { outlet }\left(\mathbf{m m}^{2}\right)\end{array}$ & $7.6 \pm 1.18$ & $7.2 \pm 1.19$ & 1.061 & 0.295 \\
\hline $\begin{array}{l}\text { Ultrasound inlet } \\
\text { outlet ratio (IOR) }\end{array}$ & $1.26 \pm 0.226$ & $0.97 \pm 0.134$ & 4.939 & $<0.001$ \\
\hline
\end{tabular}

Comparison of electrodiagnostic tests (CSI) and diagnostic ultrasound (IOR) in the patient group:

Table (4) demonstrates a comparison of electrodiagnostic tests and diagnostic ultrasound in patients with symptoms as well as signs suggestive of early cases of CTS, which revealed that CSI is more sensitive and has higher accuracy than IOR.

Table (4): Comparison of electrodiagnostic tests (CSI) and diagnostic ultrasound (IOR)

\begin{tabular}{|l|c|c|c|c|c|c|}
\hline & Cutoff & Sens. & Spec. & $\begin{array}{c}\text { PP } \\
\text { V }\end{array}$ & NPV & Accuracy \\
\hline $\begin{array}{l}\text { NVCS combined } \\
\text { sensory index (CSI) }\end{array}$ & $>0.8$ & 85.00 & 100.00 & $\begin{array}{c}100 \\
.00\end{array}$ & 87.0 & $97.5 \%$ \\
\hline $\begin{array}{l}\text { Ultrasound inlet } \\
\text { outlet ratio (IOR) }\end{array}$ & $>1$ & 80.00 & 70.00 & 72. & 77.8 & $85 \%$ \\
\hline
\end{tabular}

Combining electrodiagnostic test (CSI) as well as diagnostic ultrasound (IOR):

Combining CSI test as well as diagnostic ultrasound (IOR), the findings indicated elevated substantial differences between both groups (P <0.001), with sensitivity elevated to 100\%, excellent NPV, and good PPV (Table 5).

Table (5): Combination of CSI as well as IOR in the patient group.

\begin{tabular}{|c|c|c|c|c|c|c|c|c|}
\hline \multirow{3}{*}{ CSI and IOR } & \multicolumn{6}{|c|}{ Groups } & \multirow{2}{*}{\multicolumn{2}{|c|}{ Chi-Square }} \\
\hline & \multicolumn{2}{|c|}{ Patients } & \multicolumn{2}{|c|}{ Controls } & \multicolumn{2}{|c|}{ Total } & & \\
\hline & $\mathbf{N}$ & $\%$ & $\mathbf{N}$ & $\%$ & $\mathbf{N}$ & $\%$ & $\mathbf{X}^{2}$ & P-value \\
\hline Negative & 0 & 0.00 & 14 & 70.00 & 14 & 35.00 & \multirow{3}{*}{21.538} & \multirow{3}{*}{$<0.001 *$} \\
\hline Positive & 20 & 100.00 & 6 & 30.00 & 26 & 65.00 & & \\
\hline Total & 20 & 100.00 & 20 & 100.00 & 40 & 100.00 & & \\
\hline \multicolumn{9}{|c|}{ ROC } \\
\hline Sensitivity & \multicolumn{5}{|c|}{ NPV } & \multicolumn{3}{|l|}{ PPV } \\
\hline 100 & \multicolumn{5}{|c|}{100} & \multicolumn{3}{|l|}{76.92} \\
\hline
\end{tabular}




\section{DISCUSSION}

Conventional diagnosis of CTS based on clinical evaluation as well as electro diagnostic test which has been considered a sufficient approach to diagnosing most of the CTS cases. Sensory comparison tests for the median nerve are recommended in early stages when clinical examination is insufficient for diagnosis. Comparative studies using patient nerves as references are believed to be more sensitive ${ }^{(2)}$. The reliability as well as the sensitivity of CSI in diagnosing CTS diagnosis was further enhanced by the study of Robinson et al. ${ }^{(3)}$.

Over years, ultrasonography has been used in order to contribute to the diagnose of CTS, particularly in these cases when the compatible symptoms are detected along with normal physical as well as electrodiagnostics ${ }^{(6)}$.

In our study paresthesia and nocturnal paresthesia in lateral three and half fingers were found in all patients $(100 \%)$. The compression as well as Phalen test were positive in approximately $60 \%$ of cases whereas the Tinel test was positive in around $40 \%$ of cases ${ }^{(7,8)}$. This is in agreement with $\mathbf{M a} \mathbf{K i m}^{(9)}$ who prospectively studied patients diagnosed with CTS and a control group of asymptomatic persons. He found that Phalen's and compression tests are more sensitive than Tinel test in detection of CTS. Utilizing only these clinical maneuvers for diagnosing CTS or rule out is not recommended nor supported.

With respect to electrodiagnostic tests: Comparing patients as well as controls with regard to ring difference test, CSI, as well as thumb difference test, and elevated significant differences $(\mathrm{P}<0.001)$ were detected between both groups while the tow group demonstrated non-significant differences $(\mathrm{P}>0.05)$ regarding Palm difference. Our results partially agreed with Robinson et al. ${ }^{(3)}$ study who found that the three latency differences and the CSI findings in CTS patients were substantially different from controls $(\mathrm{P}<0.001)$. This may be because they conduct their study on a larger number of patients with moderate cases of CTS not only early or suspected cases as in the present study. One of the major findings of this study is that CSI was the most precise, specific and accurate test in early detection of CTS with a sensitivity of $85 \%$, specificity of $100 \%$, excellent PPV of $100 \%$, good NPV of $87 \%$ and accuracy of $97.5 \%$. In CTS, the large myelinated fibers are higher in sensory fiber of the median nerve than in the motor fiber and this is the cause why make the sensory fiber is more sensitive to ischemia and its affection in early stage of CTS than the motor fiber. In electrodiagnostics, studies of anomalies in the sensory fibers alone suggest early to mild CTS degree while the affection of the motor fibers increases theses degrees to mild or severe level of CTS ${ }^{(2)}$. The most sensitive tests are those of comparative studies of the median sensory fibers indicated in the event that the regular electrodiagnostics are normal, nonetheless, clinical suspicion still exists ${ }^{(2)}$. This comes in agreement with
Robinson et al. ${ }^{\left({ }^{(6)}\right.}$ who found that the CSI sensitivity was $83.1 \%$ with a specificity of $100 \%$, with a cutoff value of $\leq 1 \mathrm{~ms}$ in comparison to the single sensory test. Moreover, Lew et al. ${ }^{(\mathbf{1 0})}$ reported that CSI is a reliable method and accurate test for CTS diagnosis.

With respect to the ultrasonographic findings: The Comparison between patients as well as the controls showed statistically substantial differences between both groups $(\mathrm{P}<0.001)$ as regard CSA of median nerve at the inlet of the tunnel and Inlet-tooutlet ratio (IOR) of the median nerve cross-sectional area, however, no substantial differences were detected between both groups $(\mathrm{P}>0.05)$ with regard to median nerve CSA at the outlet of the tunnel. Our results come in agreement with $\mathbf{F u}$ et al. ${ }^{(5)}$. Their results demonstrated elevated substantial differences between patients as well as controls $(\mathrm{P}<0.001)$ as regard CSA at tunnel inlet and IOR and non-significant differences ( $>0.05$ ) regarding comparison of median nerve CSA at the outlet of the tunnel.

On the contrary, other studies have detected substantial differences in o of theutlet CSA median nerve between patients as well as controls $(\mathrm{P}<0.001)$ as in Buchberger et al. ${ }^{(11)}$ and Nakamichi et al. ${ }^{(12)}$. A possible explanation for this discrepancy could be that diagnostic ultrasonography is an operator-based tools as well as median nerve CSA measurements at the outlet of the carpal tunnel are more technically complex compared to the inlet ${ }^{(4)}$. In addition, this may be because they conduct their study on a larger number of patients with moderate cases of CTS not only early cases as our study.

In the present study, (IOR) was utilized to compensate the CSA inter the median nerve individual variability and give a more precise CTS diagnosis. In addition, our results revealed that IOR was more sensitive than CSA inlet.

The current results come in accordance with $\mathbf{F u}$ et al. ${ }^{(5)}$ who found that IOR was more efficient than CSA inlet in diagnosing CTS $(\mathrm{P}<0.001)$ with a sensitivity of $91 \%$ as well as a specificity of $93 \%$. Our results are inconsistent with Mauro et al. ${ }^{(13)}$ who found that CSA inlet sensitivity was $85.7 \%$, specificity of $55 \%$ and accuracy of $83 \%$. While IOR sensitivity was $77.9 \%$, specificity of $55.5 \%$ and accuracy of $85 \%$. Moreover, El Miedany et al. ${ }^{(14)}$, who conducted a case-control study on 78 patients and 78 normal controls, when comparing between patients as well as controls in relation to median nerve CSA at the inlet of tunnel found greater sensitivity as well as accuracy. This may be because the study did not involve CTS early stages.

For comparing the combined sensory index test (CSI) as well its sensitivity with diagnostic ultrasonography in carpal tunnel syndrome early detection.

One of the main findings of our study showed that CSI has higher sensitivity, specificity and accuracy than IOR. This agrees with Arnaldo et al. ${ }^{(15)}$ who evaluated the sensitivity of ultrasonography and electromyography in the diagnosis of carpal tunnel 
syndrome (CTS). They found that NCS sensitivity for the diagnosis of CTS was significantly more elevated than US sensitivity when assessed separately.

This is inconsistent with Fowler et al. ${ }^{(16)}$ who compared the accuracy, specificity and sensitivity of ultrasound as well as electro-diagnostic study. They found that ultrasound and NCS had a similar sensitivity, specificity and accuracy, and this may be due to their utilization of the usual way of diagnosis by US (CSA inlet only) and NCS sensory and motor tests not IOR or CSI as in the present study. In addition, they conduct their study on moderate to severe CTS cases not only early cases as our study.

The result of the combination between CSI and diagnostic ultrasound (IOR) demonstrated elevated substantial differences between patients and controls $(\mathrm{P}<0.001)$, and both tests together showed a higher sensitivity of $100 \%$.

This is compatible with the findings of Nakamichi and Tachibana (17) who examined 414 hands with clinically diagnosed idiopathic CTS and 408 normal hands as controls. It was found that the sensitivity was elevated due to the combination between both tests. Although they used different NCS and US ways for diagnosis but they reached to the same conclusion of our study that US is a complementary diagnostic test to NCS. US alone is not adequate for CTS. This is consistent with the findings of Mauro et al. ${ }^{(18)}$, who demonstrated that NCS sensitivity for the diagnosis of CTS (67.1\%) was more elevated than US sensitivity when evaluated independently. However, sensitivity increased to $76.5 \%$ after the use of both test together. The current results are also compatible with the findings of Arnaldo et al. ${ }^{(15)}$ who evaluated the sensitivity of ultrasonography as well as electromyography in CTS diagnosis, who included 70 hands with CTS clinical diagnosis. They found that NCS sensitivity was (98.6\%) which is substantially more elevated than US sensitivity (97.1\%) when assessed separately. After combining NCS and US sensitivity increased up to $100 \%$.

The present study has some limitations such as the small sample size, in addition to the absence of longterm NCS and US follow-up beyond one year. Therefore, we recommend the use of CSI in early or suspected cases of CTS in combination with US on the median nerve IOR.

\section{CONCLUSION}

It could be concluded that CSI is indicated in suspected CTS early cases with greater sensitivity as well as accuracy compared to diagnostic ultrasonography IOR on the median nerve. In contrast, diagnostic ultrasonography can detect the anatomical abnormalities of the median nerve while the physiological abnormalities of the median nerve as well as their level can be examined by NCS, consequently they are complementary tests for CTS diagnosis.
Financial support and sponsorship: Nil.

\section{Conflict of interest: Nil.}

REFERENCES

1. Lee W, Liao Y, Wei S et al. (2012): How to make electrodiagnosis of carpal tunnel syndrome with normal distal conductions? Journal of Clinical Neurophysiology, 28(1): 45-50.

2. Lawrence $R$, Robinson $L$, Micklesen $P$ et al. (2014): Optimizing the number of tests for carpal tunnel syndrome. Muscle Nerve, 23:1880-1882.

3. Robinson L, Mickelsen P, Wang $L$ (1998): Strategies for analyzing Nerve conduction data: superiority of a summary index over single tests. Muscle Nerve, 21:1166-1171.

4. Visser L, Smidt M, Lee M (2008): High-resolution sonography versus EMG in the diagnosis of carpal tunnel syndrome. J Neurol Neurosurg Psychiatry, 79:63-67.

5. Fu T, Cao M, Liu F et al. (2015): Carpal tunnel syndrome assessment with ultrasonography: value of inlet-to-outlet median nerve area ratio in patients versus healthy volunteers. PLoS One, 10(1):1-6.

6. Rahmani M, Ghasemi-Esfe A, Vaziri-Bozorg S et al. (2011): The ultrasonographic correlates of carpal tunnel syndrome in patients with normal electrodiagnostic tests. La Radiologia Medica, 116(3):489-96.

7. Phalen G (1966): The carpal tunnel syndrome: Seventeen years' experience in diagnosis and treatment of the six hundred fifty-four hands. Journal of Bone and Joint Surgery, 48: 211-228.

8. Kushner S, Ebramzadeh E, Johnson D et al. (1992): Tinel's sign and Phalen's test in carpal tunnel syndrome. Orthopedics, 15(11):1297-1302.

9. Ma H, Kim I (2012): The Diagnostic Assessment of Hand Elevation Test in Carpal Tunnel Syndrome. J Korean Neurosurg Soc., 52(5): 472-475.

10. Lew H, Wang L, Robinson L (2000): Test-retest reliability of combined sensory index: implications for diagnosing carpal tunnel syndrome. Muscle Nerve, 23: 1261-1264.

11. Buchberger W, Judmaier W, Birbamer G et al. (1992): Carpal tunnel syndrome: diagnosis with high resolution sonography. American Journal of Radiology, 159: 793-798.

12. Nakamichi K, Tachibana S (1998): Distance between the median nerve and ulnar neurovascular bundle: clinical significance with ultrasonographical assisted carpal tunnel release. J Hand Surg., 23: 870-874.

13. Mauro M, Georgios F, Adriana G et al. (2008): Diagnostic utility of ultrasonography versus nerve conduction studies in mild carpal tunnel syndrome. Arthritis \& Rheumatism: Arthritis Care \& Research, 59(3):357-366.

14. El Miedany Y, El Gaafary M, Youssef S et al. (2015): Ultrasound assessment of the median nerve: a biomarker that can help in setting a treat to target approach tailored for carpal tunnel syndrome patients. Springer Plus, 4: 13-18.

15. Arnaldo G, Bruno F, Marcelo A et al. (2014): Comparative study between physical examination, electroneuromyography and ultrasonography in diagnosing carpal tunnel syndrome. Rev Bras Ortop., 49(5): 446-451.

16. Fowler J, Munsch M, Tosti R et al. (2014): Comparison of ultrasound and electrodiagnostic testing for diagnosis of carpal tunnel syndrome: study using a validated clinical tool as the reference standard. J Bone Joint Surg Am., 96(17):148-152.

17. Nakamichi K, Tachibana S (2002): Ultrasonographic measurement of median nerve cross-sectional area in idiopathic carpal tunnel syndrome: diagnostic accuracy. Muscle Nerve, 26: 798- 803.

18. Mauro M, Georgios F, Adriana G et al. (2008): Diagnostic Utility of Ultrasonography versus Nerve Conduction Studies in Mild Carpal Tunnel Syndrome. Arthritis \& Rheumatism: Arthritis Care \& Research, 59(3):357-366 